\title{
Remote Monitoring for Understanding Mechanisms and Prediction in Psychiatry
}

\author{
George Gillett $^{1,2} \cdot$ Kate E A Saunders ${ }^{1}$
}

Published online: 2 May 2019

(C) The Author(s) 2019

\begin{abstract}
Purpose of Review Technological advances offer the potential to better characterise the symptoms and mechanisms of psychiatric disorders. This review provides an overview of how remote monitoring might inform understanding of mood and anxiety disorders, and may be used by clinicians to predict clinical outcomes and treatment response. The review also discusses salient ethical issues and the field's future directions.

Recent Findings A range of psychological and physiological remotely-collected data have been associated with clinical symptoms and outcomes, including momentary self-report, actigraphy, geolocation and heart-rate variability. Remote monitoring has shown promise in phenotyping, predicting symptoms and clinical severity, predicting treatment response and has informed psychological models of anxiety disorders.

Summary Remote monitoring is a diverse field involving the collection of clinically relevant data in naturalistic settings. Although facing significant practical and ethical challenges, it has shown much potential in developing understanding in the context of mood and anxiety disorders.
\end{abstract}

Keywords Remote monitoring · Digital phenotyping · Digital psychiatry $\cdot$ Ecological momentary assessment $\cdot$ Experience sampling methods $\cdot$ Actigraphy $\cdot$ Heart-rate variability $\cdot$ Geolocation $\cdot$ Anxiety $\cdot$ Mood disorders

\section{Introduction}

Traditional clinical assessment of psychiatric disorders has been limited by recall and symptom-state bias, while experimental assessment has been criticised for being crosssectional in nature and subject to significant interrater variability. This is particularly problematic in the context of mood and anxiety disorders where symptoms are known to be episodic, fluctuating and context-specific. These limitations have significantly impeded the understanding of the mechanisms of psychiatric illness and the development of effective

This article is part of the Topical Collection on Mood and Anxiety Disorders

George Gillett

George.gillett@medsci.ox.ac.uk

1 Department of PsychiatryWarneford Hospital University of Oxford, Oxford OX3 7JX, UK

2 Oxford University Clinical Academic Graduate School, John Radcliffe Hospital (Main Hospital), Room 3A31, The Cairns Library IT Corridor Level 3, Oxford OX3 9DU, UK treatments. The transdiagnostic nature of many symptoms poses an additional question, as it remains unclear whether different disorders share common symptoms or whether these common symptoms arise from inaccuracies and a lack of sensitivity in diagnostic categorisation.

The rapid evolution of mobile and wearable technology in the last decade, the ubiquity of mobile networks and high rates of smartphone ownership have offered new opportunities to address the challenge of traditional diagnostic techniques. Smartphones enable the collection of prospective, high frequency self-report data about symptoms in ecologically valid settings while the wide range of sensors within smartphones enable collection of passive data such as movement, light exposure and temperature. High engagement with smartphones also offers potential to quantify the use of applications, phone-calls and messaging as a proxy for symptomology. Wearable technologies such as wrist worn actigraphs and mobile ECGs devices provide additional data which can complement symptom monitoring. All these routes of digital remote monitoring therefore offer the possibility of capturing signals more representative of the underlying physiology of a disorder in comparison to traditional clinical 
assessment, offering potential to understand mechanisms of disease in psychiatry.

Here we will review the current literature on the use of digital remote techniques to inform our understanding of mechanisms and prediction in psychiatry.

\section{Digital Phenotyping}

Digital phenotyping is defined as moment-by-moment quantification of the individual-level human phenotype in situ using data from personal digital devices [1]. Data can be collected actively, for example using self-report questionnaires, or passively, for example movement data collected from a smartphone. Self-report data collected online or via mobile phones are widely used in clinical and research settings and has been shown to correlate with clinician rated depressive and manic symptoms on the HDRS-17 and YMRS $[2,3 \cdot \bullet]$. The resolution and temporal nature of such data can offer an insight into the mechanisms of psychiatric disorders.

In bipolar disorder particularly, high frequency prospective mood monitoring such as that conducted using the TrueColours [4•] system gives a richer, more accurate phenotype than conventional descriptions (Fig. 1). It has revealed a complex picture in which chronic mood variation - mood instability - is more typical than the discrete episodes which form part of the diagnostic criteria. Moreover, self-reported mood instability is common in the population (14\%) and a risk factor for bipolar disorder [5].

The nature of the nature of mood instability also appears to be diagnostically specific. In the AMoSS study [6*•] participants with bipolar disorder or borderline personality disorder and healthy volunteers reported their mood on a daily basis using a smartphone app. The three groups could be clearly differentiated on the degree of mood instability as well as the way in which different mood states related to one another.

The temporal relationship between different symptoms have also been described while phenotyping other diagnostic groups. Van Voorhees 2018 [7] employed monitoring using handheld devices to demonstrate that PTSD symptoms predicted subsequent irritability and hostile affect, but that the inverse was not true. These findings suggest that trauma history and symptoms should be implicated in anger management in this patient group. Employing similar methods, Simons 2018 found that PTSD symptoms predict conduct problems the following day in the context of alcohol dependence [8] and Short 2018 demonstrated the effect of maladaptive strategies such as avoidance on PTSD symptoms the following day [9]. In panic disorder HelbigLang 2012 used similar temporal analysis to investigate the relationship between anticipatory anxiety and panic attacks in panic disorder and agoraphobia. While panic attacks increased anticipatory anxiety, heightened anticipatory anxiety did not precede panic attacks, contrary to cognitive models of panic [10].

\section{Passive Correlates of Mood States}

The rapid expansion in wearable technologies and smartphone sensors has enabled the collection of data streams that do not require any active input from users. This passive data provides information about a range of symptoms and behaviours can be used to predict current mood state. Phone use has been explored in a number of studies as social interaction is commonly affected by mood state. In bipolar disorder significant correlations have been reported between the length and number of outgoing phone calls, number of outgoing text messages per day and symptoms of mania [11]. In bipolar depression Beiwinkel et al. found that the number of outgoing texts negatively correlated with depressive symptoms on the Hamilton Depression Rating Scale although a reversal of this correlation was not observed with the Young Mania Rating Scale score [12]. Changes in voice and speech are consistently reported as correlating with mood states in the context of structured interview calls [13] as well as clinical interactions and unstructured voice data $[14,15]$.

Activity as measured using accelerometers have revealed similarly mixed results with some studies reporting positive correlations between activity and mood while others have failed to find any significant relationship [16]. Geolocation parameters have also been explored in the context of mood disorders. Palmius et al. were able to predict current depressive symptoms with an accuracy of over $86 \%$ in individuals with bipolar disorder while Faurholt-Jepsen et al. reported significant positive correlations between cell tower ID changes per day and both rating scale scores (YMRS and HDRS17), with depressive symptoms being associated with reduced movement and manic symptoms associated with increased movement $[17 \bullet \bullet, 18]$. However, the direction of causality remains unclear.

The chronic relapsing and remitting nature of psychiatric disorders is such that many researchers have sought to use remote monitoring to predict future mood states with a view to enabling earlier initiation of preventative treatment. A variety of mathematical approaches have been used including relaxation oscillatory models [19], control charts [20] and rough paths theory [21] with varying results. It seems likely that for any model to gain the necessary accuracy upon which to base treatment decisions, predictions would need to be made at in individual rather than group level. 
Fig. 1 example of a mood chart from an individual with bipolar disorder. Red lines denote Altman Mania Rating Scale scores, blue lines denote Quick Inventory of Depression Scale scores

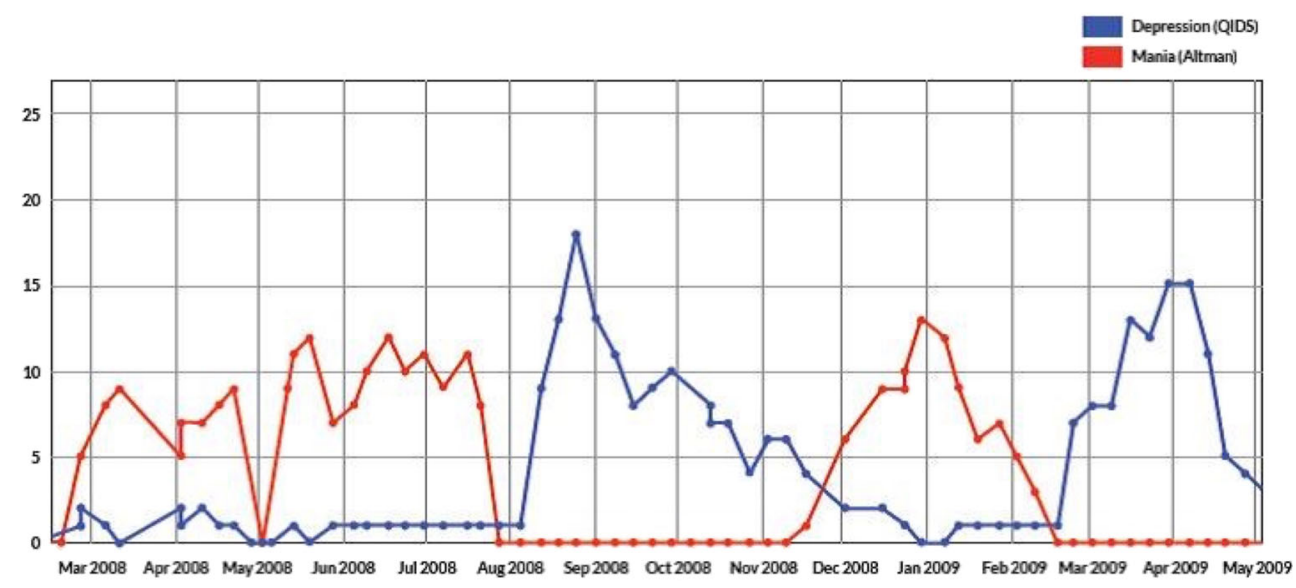

\section{Indication of Underlying Mechanisms}

The combination of self-reported mood data and data derived from wearables is beginning to provide new insights about the underlying mechanisms in psychiatric disorders. The combination of actigraphy and heart rate data collected via wearable devices have revealed a specific circadian phenotype in borderline personality disorder (BPD). Individuals with BPD had clear evidence of phase delay in the diurnal rhythm of their activity, sleep and heart rate when compared with bipolar disorder and healthy controls. Inspection of correlation coefficients controlling for gender and employment status revealed strong associations between sleep/circadian rhythm parameters and symptoms of impulsivity and mood instability in BPD which were not seen in the other groups. The findings suggest that disturbances initiating/maintaining sleep may be an exacerbating factor for borderline psychopathology and that stabilisation and consolidation of rest-activity rhythms might be considered a treatment target [22-24]. In depressed patients the measurement of HRV as an index of vagal function have been proposed as a mediator of the relationship between low mood and cardiovascular disease [25].

Remote monitoring also provides insight into the psychological mechanisms of mood and anxiety disorders. Using ecological momentary assessment (EMA) methods, Kashdan 2014 demonstrated an association between momentary experiential avoidance and anxiety symptoms during social interactions in naturalistic settings, highest among participants with SAD [26]. An EMA study by Wenze 2018 provided further insight into this association, using lag analyses to demonstrate a bidirectional relationship between experiential avoidance and negative mood, thoughts and stress [27].

Thompson 2017 used handheld electronic devices to investigate the role of should affect - the belief of how one should feel in the moment - in major depressive disorder and generalised anxiety disorder (GAD) in naturalistic settings [28]. In both disorders, participants reported higher levels of should positive affect and lower levels of should negative affect than healthy controls, even once mean affect was accounted for. Kircanski 2015 used experience sampling methods to demonstrate prevalent rumination in participants with depression and anxiety [29], and Ruscio 2015 used EMA methods to demonstrate that individuals reported more rumination after stressful events compared to controls, even after the stressfulness of an event had been adjusted for, and that rumination predicted future symptoms of anxiety [30].

As well as psychological mechanisms, remote monitoring offers insight into how behavioural factors interact with anxiety disorders. Farmer 2015 studied the effect of stressful social events over a period of 2 weeks in participants with SAD and found increases in negative affect and decreases in positive affect and self-esteem on days with more stressful social events, and that this sensitivity to stress was more marked compared to a group of healthy controls [31]. Likewise, Morgan 2017 assessed youth with SAD, GAD and/or separation anxiety and found them more sensitive to positive events and rewarding social interactions than healthy controls, experiencing an altered positive affect profile in response to positive events, informing cognitive and behavioural theories of anxiety [32].

\section{Applications in Treatment Development}

The use of remote monitoring in combination with existing treatments of known efficacy may also enable greater understanding of underlying disease mechanisms as well as predictors of therapeutic response. One of the major barriers to new drug development in psychiatry is the lack of well validated early markers of treatment response which means that treatment trials are lengthy and prohibitively expensive to run. The use of existing treatments to generate early markers of treat- 
ment response has already been hugely successful in depression. Using the Emotional Test Battery Harmer et al. have shown that early changes in negative affective bias predict subsequent improvement in mood [33]. This is now widely accepted as providing an early marker of (and cognitive model for) antidepressant efficacy using short-term drug administration in healthy volunteers and patient groups. The use of remote monitoring in this context may enable experimental models for other diagnoses to be generated. The OxLith trial is using lithium to identify early clinic, neurocognitive and biological effects of treatment as captured using remote monitoring in combination with a range of imaging modalities [34].

\section{Ethics}

Digital remote monitoring also raises a number of ethical concerns [35]. Of particular interest is respect for privacy and autonomy, especially with regard to technologies that involve long-term monitoring and aim to predict or detect illness relapse. For instance, it may be difficult to safeguard autonomy when collecting data for use in machine learning techniques where the use of the data is not hypothesis-driven and may not be clear at time of collection. There is also the possibility that self-monitoring may encourage coercion or exacerbate power relationships between service providers and users.

A related ethical concern is that remote monitoring may affect participants' identity and encourage medicalisation of everyday experiences. For instance, a qualitative study highlighted that participants worry that reactive changes in mood may be misinterpreted as illness relapse, while others raised concerns that users themselves may become preoccupied with collecting self-report data in naturalistic settings [36•].

Similarly, the collection and storage of large volumes of data involves risks relating to confidentiality and data security, especially when data is collected which is not typically understood to be medical in nature. Other potential harms include the risk that remote monitoring may increase stigma or discrimination against certain groups of people, especially those with mental illness. Not only may wearable devices attract stigma, but the use of data and modelling relating to prediction when used by third parties such as commercial enterprise or government may result in discrimination against those with mental disorders [37•]. Likewise, although engagement with smartphones and wearables is growing, there is concern that advances may not be shared equally due to individuals' limited access to technology.

The use of remote monitoring also raises the possibility of medical harm. The lack of regulation and validation of many commercially available remote monitoring methods is especially relevant in this regard [38]. Participants might be provided with poor information, or encouraged towards selfdiagnosis and self-treatment rather seeking appropriate support [39]. Equally concerning are suggestions that when used inappropriately, remote interventions may even increase symptoms of anxiety/mental distress [40]. Although remote monitoring offers a wealth of data to be collected, it is imperative that such data is validated and shown to be clinically significant before broader conclusions are drawn from it.

\section{Future Directions}

The use of remote monitoring has expanded rapidly in recent years and there are an abundance of publicly and commercially available applications and wearables. While these are popular among consumers significant concerns have been raised about their validity and safety. Nicholas et al. reported that none of the symptom apps that they identified had been subject to rigorous research or cited published material and only a small proportion had a privacy policy (Nicholas et al. 2015). Many wearables have been shown to give inaccurate readouts of sleep and activity and none of the commercially available devices publish their analysis algorithms or allow user to access raw data. At present there is no consensus as to how remotely collected data should be reported or analysed although a set of reporting guidelines for mood data is in preparation [41]. The FDA is now in the process of developing guidance for the use of digital health medical devices and has launched a pilot precertification plan for digital health technology oversight.

There are also broader challenges for clinicians and service-users when interacting with remote monitoring. A better understanding of mechanisms and prediction informed by remotely collected data may disrupt traditional psychiatric classifications and may require changes to the phenotyping of mental disorders. This is especially true as we develop potential to collect data relating to physiology, movement, light exposure and other metrics which may not have historically been thought of as relevant to psychiatric diagnosis. Nonetheless, such shifts may promise exciting developments for prediction and treatment in psychiatric care.

\section{Compliance with Ethical Standards}

Conflict of Interest George Gillett and Kate Saunders declare no conflicts of interest relevant to this manuscript.

Human and Animal Rights and Informed Consent This article does not contain any studies with human or animal subjects performed by any of the authors. 
Open Access This article is distributed under the terms of the Creative Commons Attribution 4.0 International License (http:// creativecommons.org/licenses/by/4.0/), which permits unrestricted use, distribution, and reproduction in any medium, provided you give appropriate credit to the original author(s) and the source, provide a link to the Creative Commons license, and indicate if changes were made.

\section{References}

Papers of particular interest, published recently, have been highlighted as:

- Of importance

•- Of major importance

1. Onnela JP, Rauch SL. Harnessing smartphone-based digital phenotyping to enhance behavioral and mental health. Neuropsychopharmacology : official publication of the American College of Neuropsychopharmacology. 2016;41(7):1691-6.

2. Faurholt-Jepsen M, Ritz C, Frost M, Mikkelsen RL, Margrethe Christensen E, Bardram J, et al. Mood instability in bipolar disorder type I versus type II-continuous daily electronic self-monitoring of illness activity using smartphones. J Affect Disord. 2015;186:342-9.

3.• Faurholt-Jepsen M, Vinberg M, Frost M, Christensen EM, Bardram JE, Kessing LV. Smartphone data as an electronic biomarker of illness activity in bipolar disorder. Bipolar Disorder 2015 Nov;17(7):715-28. Study identified that both passive and active smartphone data correlates with symptoms and different affective states in patients with bipolar disorder. Highlighted that both active and passive data are likely to be clinically useful as biomarkers of illness activity.

4. Simon J, Budge K, Price J, Goodwin GM, Geddes JR. Remote mood monitoring for adults with bipolar disorder: An explorative study of compliance and impact on mental health service use and costsRemote mood monitoring for adults with bipolar disorder: An explorative study of compliance and impact on mental health service use and costs. Eur Psychiatry. 2017;45: 14-9. Paper introduces the True Colours system and assesses its compliance among patients and suitability for clinical use. True Colours has revealed mood instability to be a prevalent feature of bipolar disorder, showing how remote monitoring can inform understanding of clinical phenotype.

5. Howes OD, Lim S, Theologos G, Yung AR, Goodwin GM, McGuire P. A comprehensive review and model of putative prodromal features of bipolar affective disorder. Psychol Med. 2011;41(8):1567-77.

6.• Tsanas A, Saunders KE, Bilderbeck AC, Palmius N, Osipov M, Clifford GD, et al. Daily longitudinal self-monitoring of mood variability in bipolar disorder and borderline personality disorderDaily longitudinal self-monitoring of mood variability in bipolar disorder and borderline personality disorder. J Affect Disord. 2016;205:225-33. Paper uses remote monitoring with three clinically distinguishable groups; healthy controls, bipolar disorder and borderline personality disorder subjects. Analysis of the data was able to differentiate between bipolar disorder and borderline personality disorder groups, highlighting potential for phenotyping.

7. Van Voorhees EE, Dennis PA, Elbogen EB, Fuemmeler B, Neal LC, Calhoun PS, et al. Characterizing anger-related affect in individuals with posttraumatic stress disorder using ecological momentary assessment. Psychiatry Res. 2018;261:274-80.

8. Simons JS, Simons RM, Keith JA, Grimm KJ, Stoltenberg SF, O'Brien C, et al. PTSD symptoms and alcohol-related problems among veterans: temporal associations and vulnerability. J Abnorm Psychol. 2018;127(8):733-50.

9. Short NA, Boffa JW, Clancy K, Schmidt NB. Effects of emotion regulation strategy use in response to stressors on PTSD symptoms: an ecological momentary assessment study. J Affect Disord. 2018;230:77-83.

10. Helbig-Lang S, Lang T, Petermann F, Hoyer J. Anticipatory anxiety as a function of panic attacks and panic-related self-efficacy: an ambulatory assessment study in panic disorder. Behav Cogn Psychother. 2012;40(5):590-604.

11. Faurholt-Jepsen M, Frost M, Vinberg M, Christensen EM, Bardram JE, Kessing LV. Smartphone data as objective measures of bipolar disorder symptoms. Psychiatry Res. 2014;217(1-2):124-7.

12. Beiwinkel T, Kindermann S, Maier A, Kerl C, Moock J, Barbian G, et al. Using smartphones to monitor bipolar disorder symptoms: a pilot study. JMIR Ment Health. 2016;3(1):e2.

13. Gideon J, Provost EM, McInnis M. Mood state prediction from speech of varying acoustic quality for individuals with bipolar disorder. Proc IEEE Int Conf Acoust Speech Signal Process. 2016;2016:2359-63.

14. Karam ZN, Provost EM, Singh S, Montgomery J, Archer C, Harrington $\mathrm{G}$, et al. Ecologically valid long-term mood monitoring of individuals with bipolar disorder using speech. Proc IEEE Int Conf Acoust Speech Signal Process. 2014;2014:4858-62.

15. Guidi A, Schoentgen J, Bertschy G, Gentili C, Landini L, Scilingo EP, et al. Voice quality in patients suffering from bipolar disease. Conf Proc IEEE Eng Med Biol Soc. 2015;2015:6106-9.

16. De Crescenzo F, Economou A, Sharpley AL, Gormez A, Quested DJ. Actigraphic features of bipolar disorder: a systematic review and meta-analysis. Sleep Med Rev. 2017;33:58-69.

17.• Palmius N, Tsanas A, Saunders KEA, Bilderbeck AC, Geddes JR, Goodwin GM, et al. Detecting Bipolar Depression From Geographic Location Data. IEEE Trans Biomed Eng. 2017;64(8): 1761-71. The first study to use community based objective geolocation markers to monitor depression in bipolar disorder subjects on a significant scale. Highlights potential for the development of passive data remote monitoring tools.

18. Faurholt-Jepsen M, Vinberg M, Frost M, Debel S, Margrethe Christensen E, Bardram JE, et al. Behavioral activities collected through smartphones and the association with illness activity in bipolar disorder. Int J Methods Psychiatr Res. 2016 Dec;25(4): 309-23.

19. Bonsall MB, Geddes JR, Goodwin GM, Holmes EA. Bipolar disorder dynamics: affective instabilities, relaxation oscillations and noise. J R Soc Interface. 2015;12(112):20150670.

20. Vazquez-Montes M, Stevens R, Perera R, Saunders K, Geddes JR. Control charts for monitoring mood stability as a predictor of severe episodes in patients with bipolar disorder. International journal of bipolar disorders. 2018;6(1):7.

21. Perez Arribas I, Goodwin GM, Geddes JR, Lyons T, Saunders KEA. A signature-based machine learning model for distinguishing bipolar disorder and borderline personality disorder. Transl Psychiatry. 2018;8(1):274.

22. Carr O, Saunders KEA, Bilderbeck AC, Tsanas A, Palmius N, Geddes JR, et al. Desynchronization of diurnal rhythms in bipolar disorder and borderline personality disorder. Transl Psychiatry. 2018;8(1):79.

23. Carr O, Saunders KEA, Tsanas A, Bilderbeck AC, Palmius N, Geddes JR, et al. Variability in phase and amplitude of diurnal 
rhythms is related to variation of mood in bipolar and borderline personality disorder. Sci Rep. 2018;8(1):1649.

24. McGowan N, Goodwin GM, Bilderbeck AC, Saunders KEA. Circadian rest-activity patterns in bipolar disorder and borderline personality disorder. Under review. 2019.

25. Kemp AH, Quintana DS, Quinn CR, Hopkinson P, Harris AW. Major depressive disorder with melancholia displays robust alterations in resting state heart rate and its variability: implications for future morbidity and mortality. Front Psychol. 2014;5:1387.

26. Kashdan TB, Farmer AS. Differentiating emotions across contexts: comparing adults with and without social anxiety disorder using random, social interaction, and daily experience sampling. Emotion. 2014;14(3):629-38.

27. Wenze SJ, Gaugler TL, Sheets ES, DeCicco JM. Momentary experiential avoidance: within-person correlates, antecedents, and consequences and between-person moderators. Behav Res Ther. 2018;107:42-52.

28. Thompson RJ, Kircanski K, Gotlib IH. The grass is not as green as you think: affect evaluation in people with internalizing disorders. $J$ Affect Disord. 2016;203:233-40.

29. Kircanski K, Thompson RJ, Sorenson J, Sherdell L, Gotlib IH. Rumination and worry in daily life: examining the naturalistic validity of theoretical constructs. Clin Psychol Sci. 2015;3(6):926-39.

30. Ruscio AM, Gentes EL, Jones JD, Hallion LS, Coleman ES, Swendsen J. Rumination predicts heightened responding to stressful life events in major depressive disorder and generalized anxiety disorder. J Abnorm Psychol. 2015;124(1):17-26.

31. Farmer AS, Kashdan TB. Social anxiety and emotion regulation in daily life: spillover effects on positive and negative social events. Cogn Behav Ther. 2012;41(2):152-62.

32. Morgan JK, Lee GE, Wright AGC, Gilchrist DE, Forbes EE, McMakin DL, et al. Altered positive affect in clinically anxious youth: the role of social context and anxiety subtype. J Abnorm Child Psychol. 2017;45(7):1461-72.

33. Harmer CJ, Goodwin GM, Cowen PJ. Why do antidepressants take so long to work? A cognitive neuropsychological model of antidepressant drug action. Br J Psychiatry. 2009;195(2):102-8.

34. Saunders KE, Cipriani A, Rendell J, Attenburrow MJ, Nelissen N, Bilderbeck AC, et al. Oxford Lithium trial (OxLith) of the early affective, cognitive, neural and biochemical effects of lithium carbonate in bipolar disorder: study protocol for a randomised controlled trial. Trials. 2016;17(1):116.

35. Mittelstadt B, Fairweather B, Shaw M, McBride N. The ethical implications of personal health monitoring. International Journal of Technoethics. 2014;5(2):37-60.

36. Saunders KE, Bilderbeck AC, Panchal P, Atkinson LZ, Geddes JR, Goodwin GM. Experiences of remote mood and activity monitoring in bipolar disorder: a qualitative study. Eur Psychiatry. 2017;41:

115-21 A qualitative study revealing that remote monitoring was generally accepted by patients, while highlighting some concerns to be addressed by researchers and clinicians when testing or implementing such methods.

37. Bauer M, Glenn T, Monteith S, Bauer R, Whybrow PC, Geddes J. Ethical perspectives on recommending digital technology for patients with mental illness. International journal of bipolar disorders. 2017;5(1):6 A comprehensive summary of many of the salient ethical concerns surrounding the use of remote monitoring in psychiatry.

38. Nicholas J, Larsen ME, Proudfoot J, Christensen H. Mobile apps for bipolar disorder: a systematic review of features and content quality. J Med Internet Res. 2015;17(8):e198.

39. Cosgrove V, Gliddon E, Berk L, Grimm D, Lauder S, Dodd S, et al. Online ethics: where will the interface of mental health and the internet lead us? International journal of bipolar disorders. 2017;5(1):26.

40. Foley NM, O'Connell EP, Lehane EA, Livingstone V, Maher B, Kaimkhani S, et al. PATI: patient accessed tailored information: a pilot study to evaluate the effect on preoperative breast cancer patients of information delivered via a mobile application. Breast. 2016;30:54-8.

41. Faurholt-Jepsen M, Geddes J, Goodwin GM, Bauer M, Duffy A, Kessing L, et al. Reporting guidelines on remotely collected electronic mood data in mood disorder (eMOOD) - recommendations: In press; 2019.

Publisher's Note Springer Nature remains neutral with regard to jurisdictional claims in published maps and institutional affiliations. 